\title{
Severe Fournier's gangrene in a patient with rectal cancer: case report and literature review
}

Yu Yoshino, Kimihiko Funahashi, Rei Okada, Yasuyuki Miura, Takayuki Suzuki, Takamaru Koda, Kimihiko Yoshida, Junichi Koike, Hiroyuki Shiokawa, Mitsunori Ushigome, Tomoaki Kaneko, Yasuo Nagashima, Mayu Goto, Akiharu Kurihara and Hironori Kaneko

\begin{abstract}
Background: Fournier's gangrene in the setting of rectal cancer is rare. Treatment for Fournier's gangrene associated with rectal cancer is more complex than other cases of Fournier's gangrene. We report on a patient with severe Fournier's gangrene in the setting of locally advanced rectal cancer who was treated with a combined modality therapy.

Case presentation: A 65-year-old man presented with general fatigue and anal pain. The medical and surgical histories were unremarkable. A black spot on the perineal skin surrounded by erythema was found on physical examination, suspicious for Fournier's gangrene. Computed tomography scan showed a rectal tumor invading into the bladder (clinically T4bN2M0) and abscess formation with emphysema around the rectum. He was thus diagnosed with locally advanced rectal cancer and Fournier's gangrene with a severity index score of 12 points. We created a diverting loop colostomy of the transverse colon and performed extensive debridement of the perineum and perianal area. Fifty days later, the patient underwent radical total pelvic exenteration with sacrectomy. In addition, reconstruction of the soft tissue defect was performed using the rectus muscle, the gluteus maximus muscle, and the femoral muscle. Histopathological findings of the specimen were as follows: the tumor was a moderately adenocarcinoma with invasion to the bladder and the prostate (T4b), metastases to four resected lymph nodes (N2), and lymphovascular invasion. There were no major postoperative complications, and the patient was discharged 108 days postoperatively.
\end{abstract}

Conclusions: We report a rare case of locally invasive rectal cancer associated with Fournier's gangrene. This case highlights a usual cause of Fournier's gangrene. Physicians should be cognizant not only of the more common condition but also of the rare presentations including those associated with rectal cancer.

Keywords: Fournier's gangrene, Rectal cancer, Surgical treatment, Reconstructive surgery

Abbreviations: FG, Fournier's gangrene; CT, Computed tomography; TPE, Total pelvic exenteration; APR, Abdominoperineal resection; FGSI, Fournier's gangrene severity index

\section{Background}

Fournier's gangrene (FG) is necrotizing fasciitis and commonly begins without trauma or urinary tract disease. FG in the setting of rectal cancer is rare. A specific risk factor is rectal cancer perforation. Treatment includes extensive debridement of the areas of necrosis

* Correspondence: kingkong@med.toho-u.ac.jp

Department of General and Gastroenterological Surgery, Toho University

Omori Medical Center, 6-11-1 Omorinishi Ota-Ku, Tokyo 143-8541, Japan and the administration of broad-spectrum intravenous antibiotics. In the setting of rectal cancer, the causative rectal tumor should be removed, but the timing of this is a complex clinical decision.

We report on a patient with severe FG in the setting of locally advanced rectal cancer who was treated with a combined modality therapy. 


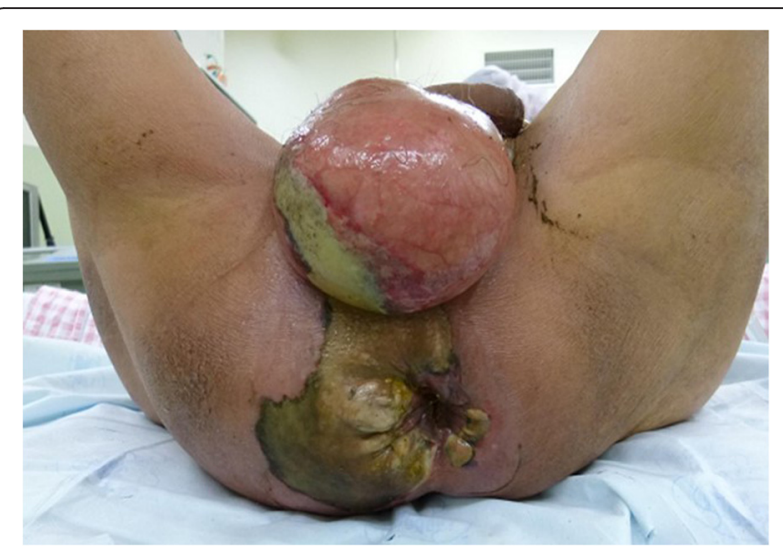

Fig. 1 The appearance of the patient's perineum. A black spot and emphysema were found in the perineal skin

\section{Case presentation}

A 65-year-old man was brought to our outpatient hospital in an ambulance complaining of general fatigue and anal pain. The medical and surgical histories were unremarkable. His initial body temperature was $95.7^{\circ} \mathrm{F}$, blood pressure was $125 / 58 \mathrm{mmHg}$, heart rate was 92 beats/min, and oxygen saturation was $98 \%$ on room air. On physical examination, a black spot on the perineal skin surrounded by erythema was found. Due to a clinical suspicion for FG, a computed tomography (CT) scan and blood tests were obtained urgently (Fig. 1). CT scan showed abscess formation with emphysema around the rectum, as well as a tumor invading the bladder (cT4b) with some lymph node metastases in the mesorectum (cN2); there was no evidence of distant metastasis (M0) (Fig. 2). Blood tests were remarkable for a hemoglobin of $3.2 \mathrm{~g} / \mathrm{dl}$ and a leukocytosis of $63,200 / \mu \mathrm{l}$. The patient was thus diagnosed with locally advanced rectal cancer and FG with a severity index score of 12 points [1]. Urgent diverting colostomy of the transverse colon and extensive debridement of the perineal area were performed (Fig. 3). Biopsies taken previously revealed a moderately differentiated adenocarcinoma.

The patient received nutritional support after surgery. Follow-up CT scan after the surgery showed no new lesions, so we performed a radical total pelvic exenteration (TPE) with sacrectomy 50 days after the initial surgery. For the urinary diversion, Bricker's operation was performed (Fig. 4). In addition, reconstruction of the soft tissue defect was performed using the rectus muscle, the gluteus maximus muscle, and the femoral muscle (Fig. 5). Histopathological findings of the specimen were as follows: the tumor was a moderate adenocarcinoma with invasion to the bladder and the prostate (T4b), metastases to four resected lymph nodes (N2), and lymphovascular invasion. There were no major postoperative complications, and the patient was discharged on postoperative day 108 .

\section{Discussion}

Although perforation of rectal cancer after treatment with bevacizumab or radiation therapy has been well documented, reports of spontaneous perforation of rectal cancer presenting as FG are rare. There were 23 cases evaluated in a review by Bruketa et al. [2]; in Japan, 17 cases including the present case have been reported (Table 1). The median age in the Japanese group was 58 (range, 30-80) years with a male to female ratio of 15:2. Six (35\%) of 17 patients had diabetes mellitus (DM) as a comorbidity. DM was considered a risk factor for FG in the Japanese group as well. Colostomy was created in 9 of 17 patients. Treatment for rectal cancer with FG was abdominoperineal resection (APR) for six patients, TPE for three patients, and chemotherapy alone for three patients. Four patients were followed conservatively without treatment. Regarding long-term prognosis of the patients, survival of 4 years and 7 months was obtained in one patient who underwent APR, and the survival of the other 16 patients was not given. In this patient, we performed TPE with sacrectomy to obtain a negative resection margin, resulting in a good oncological outcome. In addition, reconstruction was performed using the rectus muscle, the gluteus maximus muscles, and the femoral muscle. There were no major postoperative complications, and the patient was discharged on postoperative day 108.

The mortality of FG is high, frequently due to a delay in the diagnosis and management. The interesting report

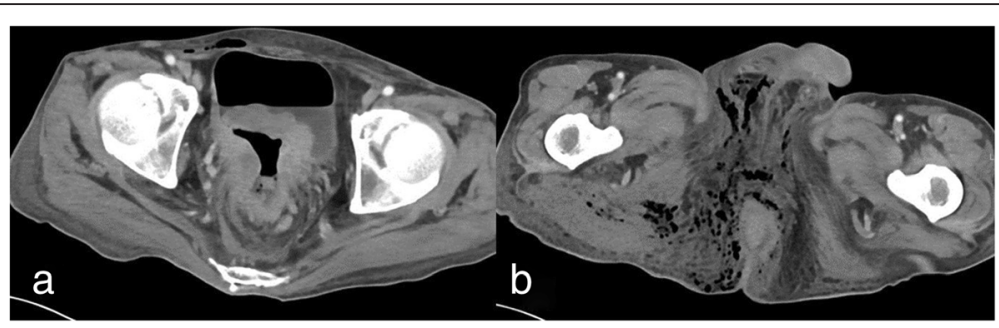

Fig. 2 Computed tomography scan showing a rectal tumor invading the bladder (a) and abscess formation with emphysema in the pelvis and perineum (b) 

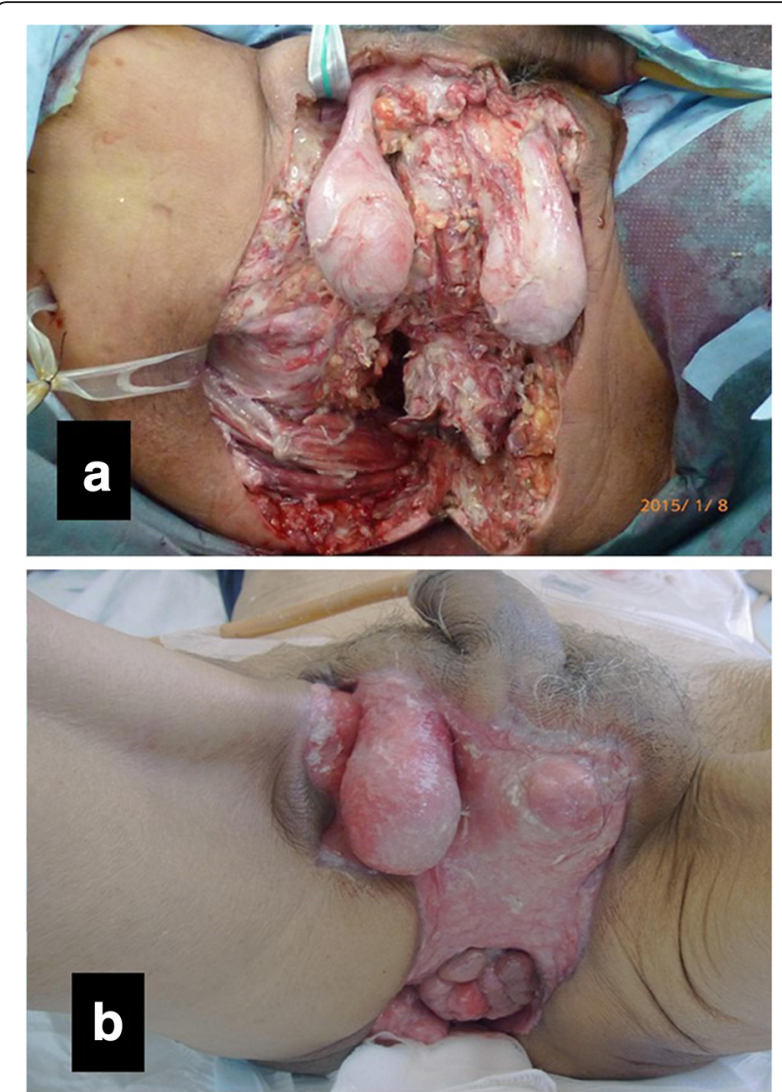

Fig. 3 Extensive debridement and diverting colostomy were performed on the day of admission (a). Fifty days later, a more extensive debridement was performed (b)

about the metabolic evaluation of FG was reported by Surucu et al. [3]. They showed the significance of ${ }^{18} \mathrm{~F}$-fluorodeoxyglucose positron emission tomography for FG in metabolic levels before the visual changes occurred. The mortality of FG in the setting of rectal cancer is not

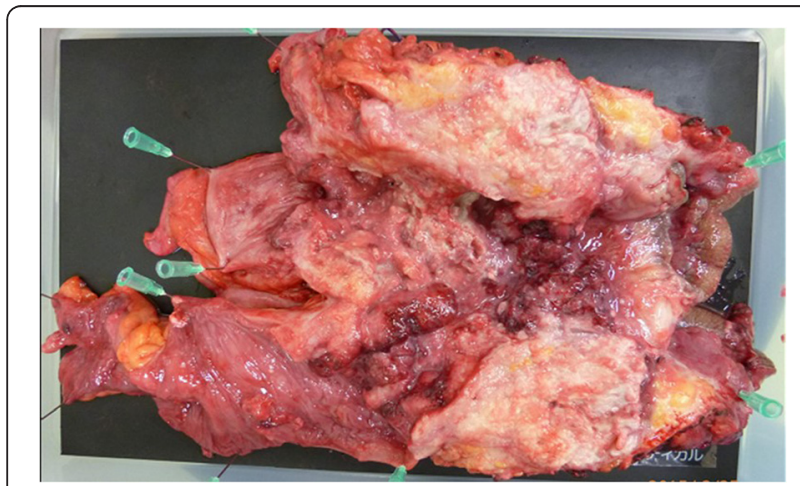

Fig. 4 TPE with sacrectomy was performed to obtain a negative resection margin. Histopathological findings of the specimen revealed a moderate adenocarcinoma invading into the bladder and the prostate (T4b), metastasis to four perirectal lymph nodes (N2), and lymphovascular invasion
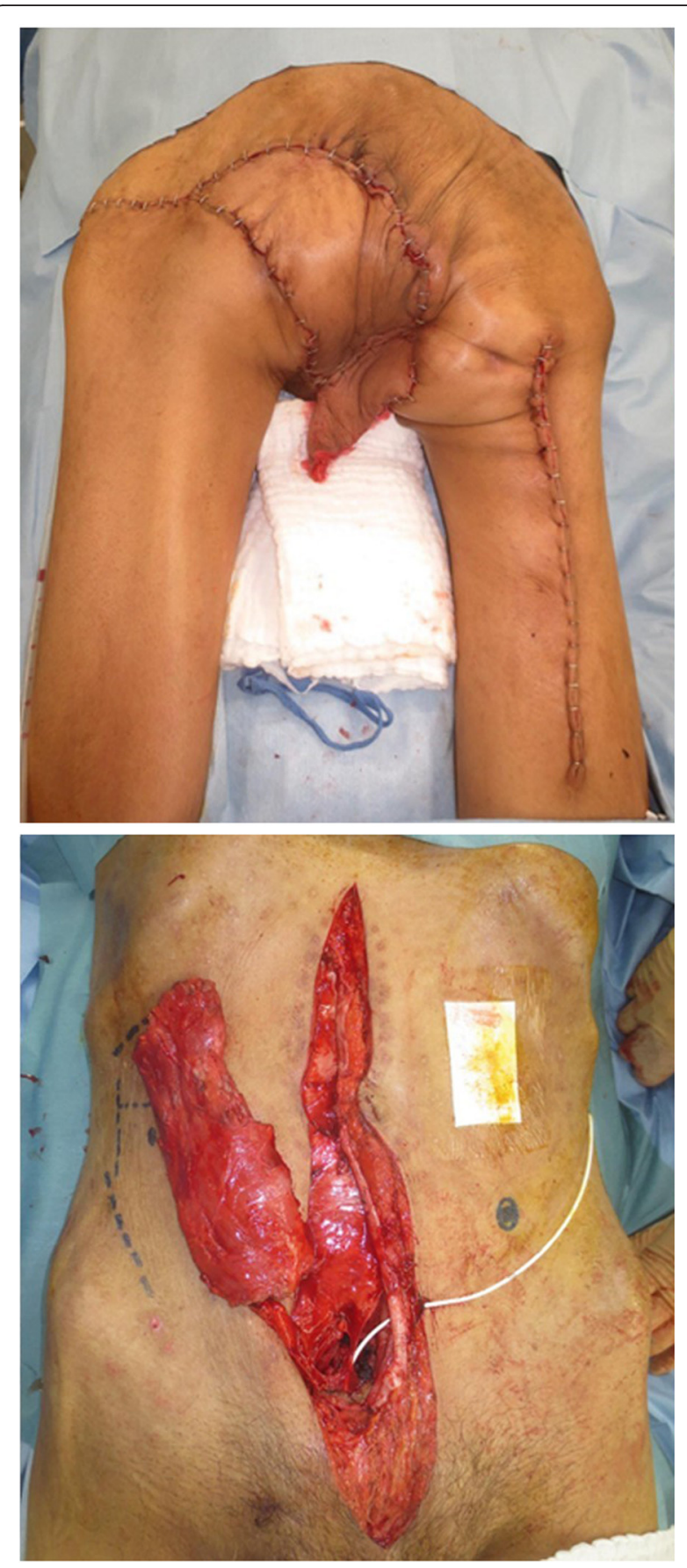

Fig. 5 Reconstruction was performed using the rectus muscle, the gluteus maximus muscle, and the femoral muscle

clear. The most common causes of death in FG are sepsis and multiple organ failure. Prognostic predictions can be based on Fournier's gangrene severity index (FGSI), comprised of the following: body temperature, heart rate, respiratory rate, and serum level of sodium, potassium, creatinine, and bicarbonate, as well as hematocrit value and leukocyte count [1]. Laor et al. [1] stated that a score of $>9$ was associated with a $75 \%$ mortality, while a score 
Table 1 Case reports of Fournier's gangrene in the setting of rectal cancer in Japan

\begin{tabular}{|c|c|c|c|c|c|c|c|c|}
\hline No. & Author & & Gender & Age & Location & Comorbidity & Operation & Outcome \\
\hline 1 & Futamura et al. [6] & 1995 & M & 56 & $\mathrm{Rb}$ & None & APR & 4 years and 7 months, alive \\
\hline 2 & Fujisawa et al. [7] & 1999 & M & 75 & $\mathrm{Rb}$ & None & None & Death at 6 days \\
\hline 3 & Nakao et al. [8] & 1999 & M & 51 & $\mathrm{Rb}$ & DM & None & Unknown \\
\hline 4 & Saito et al. [9] & 2000 & M & 60 & $\mathrm{Rb}$ & None & TPE & 1 year, alive \\
\hline 5 & Noriyuki et al. [10] & 2003 & M & 58 & Ra & DM & $5-F U+L V$ & 1 year and 2 months, alive \\
\hline 6 & Enomoto at al. [11] & 2006 & M & 35 & $\mathrm{Rb}$ & None & APR & 3 months, alive \\
\hline 7 & Moriwaki et al. [12] & 2007 & M & 30 & $\mathrm{Rb}-\mathrm{P}$ & Unknown & APR & Death at 11 months \\
\hline 8 & Kojima et al. [13] & 2007 & M & 56 & $\mathrm{Rb}$ & DM & TPE & 4 months, alive \\
\hline 9 & Morohashi et al. [14] & 2008 & M & 60 & $\mathrm{Rb}$ & $\mathrm{DM}$ & None & Death at 2 months \\
\hline 10 & Ishibashi et al. [15] & 2009 & $\mathrm{~F}$ & 80 & $\mathrm{Rb}$ & HT & APR & 2 months, alive \\
\hline 11 & Yamazaki et al. [16] & 2010 & M & $50 \mathrm{~s}$ & $\mathrm{Rb}$ & $\mathrm{DM}$ & APR & 1 months, alive \\
\hline 12 & Onizuka et al. [17] & 2010 & M & 55 & Rs & None & Chemo & 1 year and 2 months, alive \\
\hline 13 & Tanaka et al. [18] & 2010 & $\mathrm{~F}$ & 52 & $\mathrm{Rb}$ & $\mathrm{DM}$ & APR & 5 months, alive \\
\hline 14 & Watabe et al. [19] & 2013 & M & 77 & $\mathrm{Rb}-\mathrm{P}$ & Unknown & None & 2 months, alive \\
\hline 15 & Monma et al. [20] & 2013 & M & 79 & $P$ & HT & CRT & 1 year, alive \\
\hline 16 & Kawagoe et al. [21] & 2014 & M & 72 & $\mathrm{Rb}$ & None & FOLFOX + bev & 6 months, alive \\
\hline 17 & Our case & 2016 & M & 65 & $\mathrm{Rb}$ & None & TPE & Death at 1 year \\
\hline
\end{tabular}

$M$ male, $F$ female, $R b$ lower rectum, $R$ s rectosigmoid colon, $P$ proctos, $A P R$ abdominoperineal resection, $T P E$ total pelvic exenteration, $C$ hemo chemotherapy, $C R T$ chemoradiation therapy, Bev bevacizumab, DM diabetes mellitus, HT hypertension

of $\leq 9$ corresponded to a $78 \%$ probability of survival. While our patient presented with an FGSI score of 12 points, we managed to treat the patient without a major complication. Recently, hyperbaric oxygen (HBO) has been recommended as an additional therapy for patients with FG because HBO inhibits the growth of anaerobic bacteria in the affected tissues, prevents further extension of tissue necrosis, and reduces systemic toxicity $[4,5]$. Actually, for this patient creating colostomy as an initial procedure, early radical debridement of necrotic tissues, drainage, and antibiotic therapy were effective to manage severe FG in the setting of rectal cancer. Although TPE with sacrectomy was required to obtain a negative resection margin, we could get a good outcome. Although adjuvant therapy was required because of locally advanced rectal cancer of pathologically T4b
N2 M0, the patient was followed up at the outpatient section of our institution without adjuvant therapy until all wound had healed completely. Unfortunately, we suspected that local recurrence developed in the perineal wound because of an abdominal ${ }^{18} \mathrm{~F}$-fluorodeoxyglucose positron emission tomography in 5 months after curative TPE (Fig. 6). The patient died of primary disease 1 year after prior surgery.

\section{Conclusions}

Aggressive debridement, broad-spectrum antibiotics, and intensive supportive care are critical for the management of FG. Although colorectal cancer has previously been reported as a cause of FG, it remains an extremely rare phenomenon.

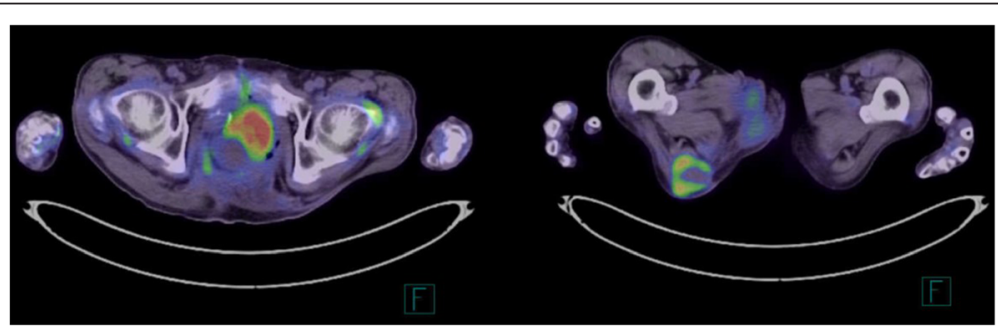

Fig. $6{ }^{18}$ F-fluorodeoxyglucose positron emission tomography scan demonstrated increased fluorodeoxyglucose uptake lesions in the perineal wound. The maximum standardized uptake values (SUV max) of the lesions were 6.36 and 13.45, respectively. Local recurrence was suspected because of an abnormal ${ }^{18} \mathrm{~F}$-fluorodeoxyglucose positron emission tomography scan 


\section{Acknowledgements}

We thank for the help of the plastic surgeons at Toho University Omori Medical Center. Without their efforts, this operation would not be possible.

\section{Funding}

The authors have no support or funding to report.

\section{Availability of data and materials}

All relevant data are within the paper.

\section{Authors' contributions}

YY, KF, RO, MY, TS, TK, JK, HS, MU, TK, and YN performed the surgery. YY and $\mathrm{KF}$ were the major contributors in writing the manuscript. MG, KY, and $A K$ helped $Y Y$ and $K F$ to collect references. HK has been involved in drafting the manuscript. All authors read and approved the final manuscript.

\section{Competing interests}

The authors declare that they have no competing interests.

\section{Consent for publication}

Written informed consent was obtained from the patient for publication of this case report and any accompanying images. A copy of the written consent is available for review by the Editor-in-Chief of this journal.

\section{Ethics approval and consent to participate}

Because this report involves no experiment, ethics approval is waived.

\section{Received: 5 February 2016 Accepted: 17 August 2016}

Published online: 01 September 2016

\section{References}

1. Laor E, Palmer LS, Tolia BM, Reid RE, Winter HI. Outcome prediction in patients with Fournier's gangrene. J Urol. 1995;154(1):89-92.

2. Bruketa T, Majerovic M, Augustin G. Rectal cancer and Fournier's gangrene-current knowledge and therapeutic options. World J Gastroenterol. 2015;21(30):9002-20. doi:10.3748/wjg.v21.i30.9002.

3. Sürücü E, Canda AE, Kaya GC, Tuna B, Sengoz T, Yorukoglu K, et al. F-18 fluorodeoxyglucose PET in Fournier gangrene. Clin Nucl Med. 2010;35(8):625-7.

4. Mallikarjuna MN, Vijayakumar A, Partil VS, Shivswamy BS. Fournier's gangrene: current practices. ISRN Surgery. 2012. doi:10.5402/2012/942437.

5. Paris VM, Santora T and Rukstalis DB. Fournier's gangrene. http://emedicine. Medscape.com/article/2028899. 16 November 2015.

6. Futamura N, Kano N, Fukuhara N, Komura Y, Miyamoto K, Maeda H, et al. A case of rectal cancer with Fournier's gangrene. In Japanese. Nippon Rinsyo Geka Igakukai Zasshi. 1995;56(2):399-402.

7. Fujisawa M, Maruyama T, Kojima K, Fukasawa M, Beppu T, Futagawa S, et al. A case of Fournier's gangrene associated with rectal cancer. In Japanese. Nippon Rinsyo Geka Gakkai Zasshi. 1999;60(6):1583-6.

8. Nakao T, Inatsugi N, Yoshikawa S, Takamura H, Masuda T, Nakano H. Fournier's gangrene caused by pentration of rectal cancer in an adult. In Japanese. Nippon Rinsyo Geka Gakkai Zasshi. 1999;60(12):3293-6.

9. Saito C, Yamada A, Kosaka K, Konno M, Toriyabe S. A case of Fournier's gangrene caused by skin perforation of rectal cancer. In Japanese. Nippon Keisei Geka Gakkai Zasshi. 2000;20(1):31-5.

10. Noriyuki T, Shimatani K, Shintaku S, lida M, Moriuchi H, Sakamoto Y, et al. A case report of advanced rectal carcinoma with Fournier's gangrene. In Japanese. Shoukakigeka. 2003:26(3):371-5.

11. Enomoto T, Sumiyama Y, Nagao J, Saida Y, Watanabe M, Asai K, et al. Development of Fournier's gangrene leading to a diagnosis of colorectal cancer. In Japanese. Nippon Gekakei Renngo Gakkai Zasshi. 2006;31(2):231-4.

12. Moriwaki Y, Yamamoto T, Anze M, Sugiyama M. A case of Fournier's gangrene due to advanced rectal cancer treated by central department including several specialists. In Japanese. Rinsyo Geka. 2007;62(6):845-8.

13. Kojima Y, Kamano T, Sakamoto K, Matsuda M, Sengoku H, Takita N, et al. A case of Fournier's gangrene caused by perforation of rectal carcinoma. In Japanese. Nippon Syoukaki Geka Gakkai Zasshi. 2007:40(4):485-90.

14. Morohashi H, Yamada K, Matsuura O, Yamazaki S, Fujita M. A case of Fournier's gangrene with rectal cancer. In Japanese. Nippon Rinsyo Geka Gakkai Zasshi. 2008;69(7):1823-7.
15. Ishibashi Y, Ito Y, Wakabayashi K. A case of Fournier's gangrene caused by penetration of rectal carcinoma. In Japanese. Nippon Rinsyo Geka Gakkai Zasshi. 2009;70(6):1772-776.

16. Yamazaki T, Goi T, Nakazawa T, Ohtsuki T, Katayama K, Yamaguchi A. A case of Fournier's gangrene with rectal cancer. In Japanese. Geka. 2010;72(10):1125-9.

17. Onitsuka K, Ito S, Tanoue T, Yamauchi M, Yamayoshi T, Kidogawa H. Three cases of Fournier's gangrene. In Japanese. Nippon Rinsyo Geka Gakkai Zasshi. 2010;71(12):3219-22.

18. Tanaka N, Tanabe A, Yamamoto J, Kusumoto K. Fournier's gangrene happened due to rectal cancer. In Japanese. Nippon Sousyo Geka Gakkai Zasshi. 2010;1 (2):102-6.

19. Watabe H, Tonouchi H, Noguchi T, Imaoka Y, Yokoe T, Ojima E, et al. A case of Fournier's gangrene caused by penetration of rectal carcinoma. In Japanese. Nippon Geka Kansensyou Gakkai Zasshi. 2013;10(1):149-53.

20. Monma T, Kikuchi D, Watanabe Y, Onozawa H, Suzuki S, Nakamura I, et al. A case of anal canal cancer which developed Fournier's syndrome after chemoradiotherapy. In Japanese. Nippon Daicho Komonbyo Gakkai Zasshi. 2013;66(5):358-63.

21. Kawagoe K, Hamasaki K, Ishikawa H, Fukuoka H, Taniguchi D, Shibuya A, et al. Management of Fournier's gangrene caused by rectal cancer for early chemotherapy. In Japanese. Nippon Daicho Komonbyo Gakkai Zasshi. 2014;67(7):448-53.

\section{Submit your next manuscript to BioMed Central and we will help you at every step:}

- We accept pre-submission inquiries

- Our selector tool helps you to find the most relevant journal

- We provide round the clock customer support

- Convenient online submission

- Thorough peer review

- Inclusion in PubMed and all major indexing services

- Maximum visibility for your research

Submit your manuscript at www.biomedcentral.com/submit
Biomed Central 